\title{
Knowledge, Attitude, and Practice Survey of Gastroparesis in Asia by Asian Neurogastroenterology and Motility Association
}

\author{
Tadayuki Oshima, ${ }^{1 *}$ Kewin T H Siah, ${ }^{2,3 *}$ Yong Sung Kim, ${ }^{4}$ Tanisa Patcharatrakul, ${ }_{1}^{5,6}$ Chien-Lin Chen, ${ }^{7,8}$ Sanjiv Mahadeva, ${ }^{9}$ \\ Hyojin Park, ${ }^{10}$ Min-Hu Chen, ${ }^{11}$ Ching-Liang Lu, ${ }^{12}$ Xiaohua Hou, ${ }^{13}$ Duc T Quach, ${ }^{14}$ Ari F Syam, ${ }^{15}$ M Masudur Rahman, ${ }^{16}$ \\ Yinglian Xiao, ${ }^{11}$ Liu Jinsong, ${ }^{13}$ Andrew S B Chua, ${ }^{17}$ and Hiroto Miwa ${ }^{1}$
}

\begin{abstract}
${ }^{\prime}$ Division of Gastroenterology and Hepatology, Department of Internal Medicine, Hyogo College of Medicine, Nishinomiya, Hyogo, Japan; ${ }^{2}$ Department of Medicine, Yong Loo Lin School of Medicine, National University of Singapore, Singapore; ${ }^{3}$ Division of Gastroenterology and Hepatology, Department of Medicine, National University Hospital, Singapore; ${ }^{4}$ Digestive Disease Research Institute, School of Medicine, Wonkwang University, Iksan, Jeollabuk-do, Korea; ${ }^{5}$ Center of Excellence in Neurogastroenterology and Motility, Faculty of Medicine, Chulalongkorn University, Bangkok, Thailand; ${ }^{6}$ Division of Gastroenterology, Department of Medicine, King Chulalongkorn Memorial Hospital, Thai Red Cross Society, Bangkok, Thailand; ' Department of Medicine, Hualien Tzu Chi Hospital, Buddhist Tzu Chi Medical Foundation and Tzu Chi University, Hualien, Taiwan; ${ }^{8}$ Institute of Medical Sciences, College of Medicine, Tzu Chi University, Hualien, Taiwan; ${ }^{9}$ Department of Medicine, University of Malaya, Kuala Lumpur, Malaysia; ${ }^{10}$ Department of Internal Medicine, Gangnam Severance Hospital, Yonsei University, Seoul, Korea; ${ }^{11}$ Department of Gastroenterology and Hepatology, First Affiliated Hospital, Sun Yat-sen University, Guangzhou, China; ${ }^{12}$ Endoscopy Center for Diagnosis and Treatment, Taipei Veterans General Hospital, Taipei, Taiwan; ${ }^{13}$ Division of Gastroenterology, Union Hospital, Tongji Medical College, Huazhong University of Science and Technology, Wuhan, China; ${ }^{14}$ Department of Internal Medicine, University of Medicine and Pharmacy at Ho Chi Minh City, Ho Chi Minh, Vietnam; ${ }^{15}$ Division of Gastroenterology, Department of Internal Medicine, Faculty of Medicine, Universitas Indonesia/Cipto Mangunkusumo Hospital, Jakarta Pusat, Indonesia; ${ }^{16}$ Department of Gastroenterology, Sheikh Russel National Gastroliver Institute and Hospital, Dhaka, Bangladesh; and ${ }^{17}$ Gastro Center Ipoh, Ipoh, Malaysia
\end{abstract}

\section{Background/Aims}

Gastroparesis is identified as a subject that is understudied in Asia. The scientific committee of the Asian Neurogastroenterology and Motility Association performed a Knowledge, Attitude, and Practices survey on gastroparesis among doctors in Asia.

\section{Methods}

The questionnaire was created and developed through a literature review of current gastroparesis works of literature by the scientific committee of Asian Neurogastroenterology and Motility Association.

\section{Results}

A total of 490 doctors from across Asia (including Bangladesh, China, Hong Kong, Indonesia, Japan, Malaysia, Myanmar, the Philippines, Singapore, South Korea, Taiwan, Thailand, and Vietnam) participated in the survey. Gastroparesis is a significant gastrointestinal condition. However, a substantial proportion of respondents was unable to give the correct definition and accurate diagnostic test. The main reason for lack of interest in diagnosing gastroparesis was "the lack of reliable diagnostic tests" (46.8\%) or "a lack of effective treatment" (41.5\%). Only $41.7 \%$ of respondents had access to gastric emptying scintigraphy. Most doctors had never diagnosed gastroparesis at all (25.2\%) or diagnosed fewer than 5 patients a year (52.1\%).

\section{Conclusions}

Gastroparesis can be challenging to diagnose due to the lack of instrument, standardized method, and paucity of research data on normative value, risk factors, and treatment studies in Asian patients. Future strategies should concentrate on how to disseminate the latest knowledge of gastroparesis in Asia. In particular, there is an urgent need to estimate the magnitude of the problems in high risk and idiopathic patients as well as a standardized diagnostic procedure in Asia.

(J Neurogastroenterol Motil 2021;27:46-54)

Key Words

Asia; Dyspepsia; Gastroparesis; Health care surveys; Health education 
Received: May 25, 2020 Revised: None Accepted: July 21, 2020

(a) This is an Open Access article distributed under the terms of the Creative Commons Attribution Non-Commercial License (http:// creativecommons. org/licenses/by-nc/4.0) which permits unrestricted non-commercial use, distribution, and reproduction in any medium, provided the original work is properly cited.

*Correspondence: Tadayuki Oshima and Kewin T H Siah are equally responsible for this work.

Tadayuki Oshima, MD

Division of Gastroenterology and Hepatology, Department of Internal Medicine, Hyogo College of Medicine, 1-1 Mukogawa-

cho, Nishinomiya, Hyogo 663-8501, Japan

Tel: +81-798-45-6662, Fax: +81-798-45-6661, E-mail: t-oshima@hyo-med.ac.jp

Kewin T H Siah, MD

Division of Gastroenterology and Hepatology, Department of Medicine, National University Hospital, NUHS Tower Block, Level

10, $1 \mathrm{E}$ Kent Ridge Road, 119228 Singapore

Tel: +65-6779-5555, Fax: +65-6775-1518, E-mail: Kewin_siah@nuhs.edu.sg

Tadayuki Oshima, Kewin T H Siah, and Yong Sung Kim contributed equally as 1st author to this work.

\section{Introduction}

Gastroparesis is a condition in which the stomach cannot empty food in a normal timely fashion. It is characterized by sluggish emptying of solid food or liquids from the stomach. Gastroparesis is characterized by a combination of cardinal symptoms (nausea, vomiting, abdominal pain, early satiety, fullness, and bloating) and no evidence of mechanical obstruction during gastroscopy, and a delayed 4-hour solid-phase gastric-emptying scan. ${ }^{1-3}$ The most common causes of gastroparesis are neuropathic disorders such as diabetes mellitus, idiopathic, post-vagotomy, and scleroderma among myopathic disorders. ${ }^{1,4}$

There is a common symptom overlap between gastroparesis and functional dyspepsia (FD). Some of the symptoms of gastroparesis, such as epigastric pain or discomfort, bloating, and early satiety, are also common symptoms in FD. ${ }^{5}$ Quartero et $\mathrm{al}^{6}$ performed a meta-analysis of disturbed solid-phase gastric emptying in $\mathrm{FD}$ and found that almost $40 \%$ of FD patients had a significant delay of gastric emptying. Clinical history alone or the use of the Gastroparesis Cardinal Symptom Index (GCSI) score was also unable to distinguish adult FD from gastroparesis. ${ }^{7} \mathrm{FD}$ may be associated with accelerated or delayed gastric emptying, impaired gastric accommodation, and heightened sensitivity in the stomach. Most patients with FD often have normal gastric emptying.

However, amongst FD patients with delayed gastric emptying, the degree of delay is usually mild (10-20\% of material remaining at 4 hours). ${ }^{8}$ The American College of Gastroenterology and American Gastroenterological Association guidelines for dyspepsia advised that a gastric emptying scintigraphy (GES) is not indicated in the routine assessment of FD patients, but can be considered for resistant FD patients with predominant nausea and vomiting symptoms. ${ }^{9}$ The Asian consensus report on FD similarly agreed that disturbed gastroduodenal motility is one of the pathophysiologic mechanisms in FD, but many studies have failed to show a correlation between delayed gastric emptying and dyspeptic symptoms. ${ }^{10}$ Asano et $\mathrm{al}^{11}$ showed that delayed emptying was present in $10.6 \%$ of FD patients in a landmark study, but there was no association between gastric motility abnormalities and dyspeptic symptoms.

Since the publication of the dyspepsia treatment guidelines, newer studies have advanced our understanding of gastroparesis, from symptomatology to potential new treatments. ${ }^{12}$ Wuestenberghs et $\mathrm{al}^{13}$ showed that there was no difference between a dyspeptic patient with or without delayed gastric emptying. However, there was an association between severely delayed gastric emptying time (half emptying time above 200 minutes) with dyspeptic symptoms (postprandial fullness, abdominal pain, bloating, and early satiety) and a lower quality of life. ${ }^{13}$

In contrast to the volume of publications in the West, there seems to be a relative lack of interest in gastroparesis research in Asia. We postulated that the lack of knowledge, confusion, and inconsistencies both in definition and investigation of gastroparesis may have led to the unwillingness of clinicians to order GES, consequently under-diagnosing gastroparesis among Asian patients. To understand the extent of this issue, we aim to perform a Knowledge, Attitude, and Practices (KAP) survey to assess the knowledge of gastroparesis among doctors in Asia, to grasp the attitude of doctors regarding suspected gastroparesis, and to identify gaps in practice among doctors. Apart from differences in diagnostic methods, this study also aims to identify discrepancies in gastroparesis management among Asian physicians. 


\section{Materials and Methods}

\section{Study Design}

An invitation to participate in the survey was sent to representative members of the Asian Neurogastroenterology and Motility Association (ANMA). All participants who agreed to participate in the study could access the questionnaire through the MySurvey application. The KAP theory-based questionnaire was created and developed through a thorough literature review of current gastroparesis works of literature by 3 gastrointestinal (GI) motility experts with experience over 15 years were selected from ANMA research committee. The questionnaire comprised 3 constructs of the KAP regarding the definition, investigation, and management of gastroparesis. For each item, the response was yes/no or unknown/uncertain. The KAP questions regarding gastroparesis are shown in Table 1. A paper-based questionnaire was also prepared for Japanese participants. The questions were translated into Japanese by one of the original planners of the questionnaire and back-translated to English for accuracy. The questions in the questionnaire are provided in Table 1 . The final questionnaire was reviewed and approved by the scientific committee of ANMA. The survey was conducted from March to July 2019 (local and international ethics approval: the National Healthcare Group DSRB reference 2019/00046 and ethics review board of Hyogo College of Medicine [No. 3120]).

\section{Statistical Methods}

All categorical data were expressed as proportions and were compared using the chi-square test. To determine the factors associated with order of GES, we estimated the odds ratio (OR) and 95\% confidence interval (CI). An unconditional logistic regression by multivariate analysis was performed to test the influence of several factors in association with order of GES. All reported $P$-values were two-sided and those less than 0.05 were considered to be statistically significant. SPSS version 22.0 (IBM Corp, Armonk, NY, USA), was used for statistical analyses.

\section{Results}

\section{Study Population}

The ANMA gastroparesis survey included 490 doctors (gastroenterologist, internist, and general surgeons) from across Asia (including Bangladesh, China, Hong Kong, Indonesia, Japan, Malaysia, Myanmar, the Philippines, Singapore, South Korea, Taiwan, Thailand, and Vietnam). The characteristics of the participants by country are shown in Table 2. Most participants were gastroenterologists $(81.2 \%)$ and experienced doctors more than 10 years after graduation from medical school (70.6\%). The male to female ratio was $3: 1$.

\section{Knowledge for Gastroparesis in Asia}

Variations existed on the definition of gastroparesis among

Table 1. The Knowledge, Attitude, and Practice Questions of Gastroparesis

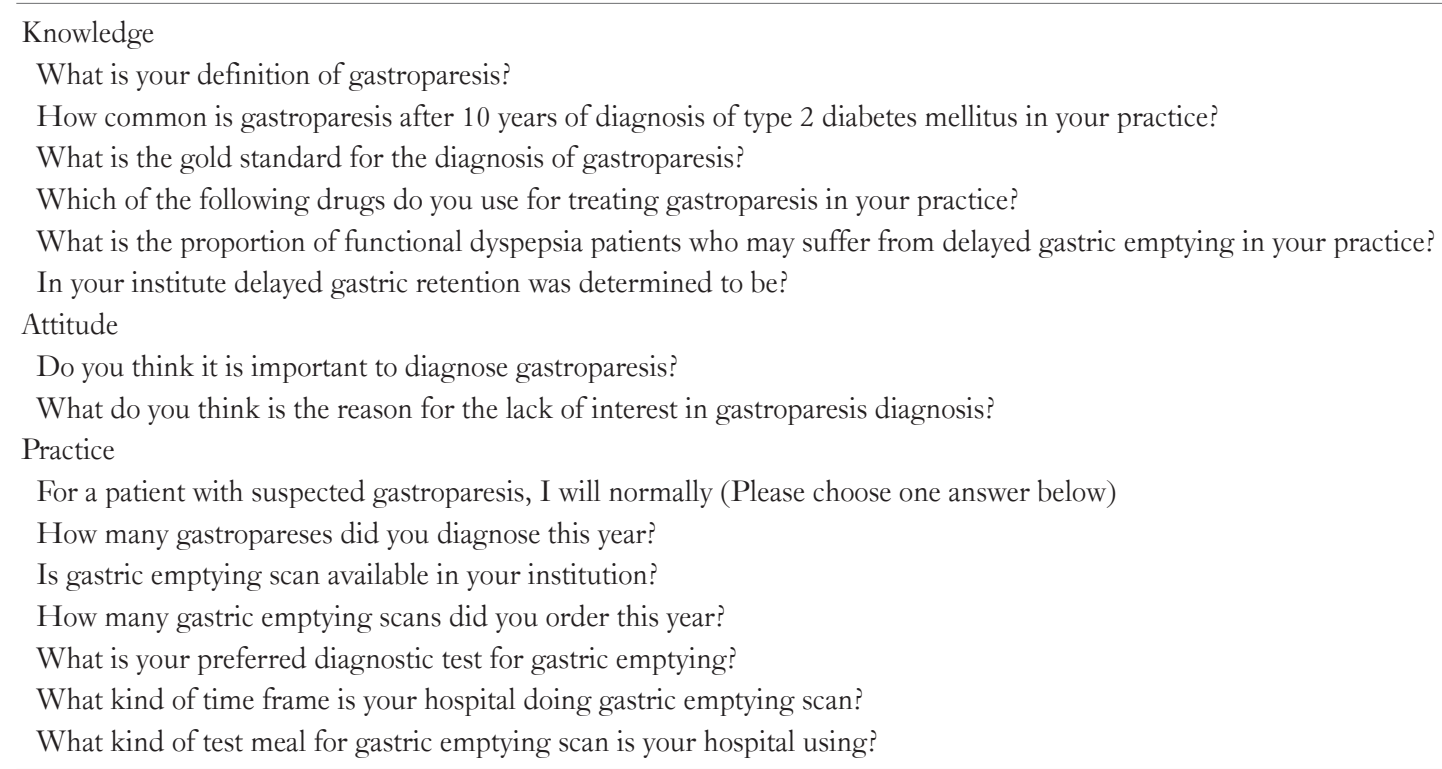


Table 2. Demographic Characteristics of Participants in the Asian Neurogastroenterology and Motility Association Gastroparesis Survey

\begin{tabular}{|c|c|c|c|c|c|c|c|c|c|c|c|c|}
\hline \multirow{2}{*}{ Country } & \multirow{2}{*}{$\mathrm{n}$} & \multicolumn{2}{|c|}{$\operatorname{Sex}(\%)$} & \multicolumn{4}{|c|}{ Occupation (\%) } & \multicolumn{4}{|c|}{ Education (\%) } & \multirow{2}{*}{$\begin{array}{l}\text { Available } \\
\text { GES (\%) }\end{array}$} \\
\hline & & Male & Female & GE & GS & GM & Other & $\leq 5 \mathrm{yr}$ & $6-10 \mathrm{yr}$ & $11-20 \mathrm{yr}$ & $>20 \mathrm{yr}$ & \\
\hline Bangladesh & 26 & 96.2 & 3.8 & 57.7 & 0.0 & 3.8 & 0.0 & 0.0 & 0.0 & 61.5 & 38.5 & 0.0 \\
\hline China & 27 & 48.1 & 51.9 & 92.6 & 0.0 & 0.0 & 7.4 & 3.7 & 11.1 & 33.3 & 51.9 & 59.3 \\
\hline Hong Kong & 2 & 50.0 & 50.0 & 50.0 & 50.0 & 0.0 & 0.0 & 0.0 & 0.0 & 0.0 & 100.0 & 100.0 \\
\hline Indonesia & 35 & 80.0 & 20.0 & 51.4 & 0.0 & 25.7 & 22.9 & 11.4 & 20.0 & 42.9 & 25.7 & 8.6 \\
\hline Japan & 133 & 91.0 & 9.0 & 86.5 & 6.0 & 3.0 & 4.5 & 0.8 & 11.3 & 42.1 & 45.9 & 15.0 \\
\hline Malaysia & 29 & 55.2 & 44.8 & 20.7 & 0.0 & 79.3 & 0.0 & 0.0 & 65.5 & 34.5 & 0.0 & 37.9 \\
\hline Myanmar & 2 & 50.0 & 50.0 & 50.0 & 50.0 & 0.0 & 0.0 & 0.0 & 0.0 & 0.0 & 100.0 & 50 \\
\hline The Philippines & 2 & 100.0 & 0.0 & 100.0 & 0.0 & 0.0 & 0.0 & 0.0 & 0.0 & 50.0 & 50.0 & 0.0 \\
\hline Singapore & 35 & 65.7 & 34.3 & 94.3 & 0.0 & 5.7 & 0.0 & 40.0 & 37.1 & 17.1 & 5.7 & 97.1 \\
\hline South Korea & 54 & 85.2 & 14.8 & 100.0 & 0.0 & 0.0 & 0.0 & 0.0 & 1.9 & 33.3 & 64.8 & 92.6 \\
\hline Taiwan & 55 & 92.7 & 7.3 & 92.7 & 0.0 & 5.5 & 1.8 & 5.5 & 32.7 & 29.1 & 32.7 & 58.2 \\
\hline Thailand & 31 & 51.6 & 48.4 & 93.5 & 6.5 & 6.5 & 0.0 & 6.5 & 35.5 & 48.4 & 9.7 & 54.8 \\
\hline Vietnam & 59 & 40.7 & 59.3 & 64.4 & 10.2 & 23.7 & 1.7 & 20.3 & 33.9 & 32.2 & 13.6 & 28.8 \\
\hline
\end{tabular}

GE, gastroenterologist; GS, general surgeon; GM, general medicine; Others, medicine subspecialty; GES, gastric emptying scintigraphy.

A

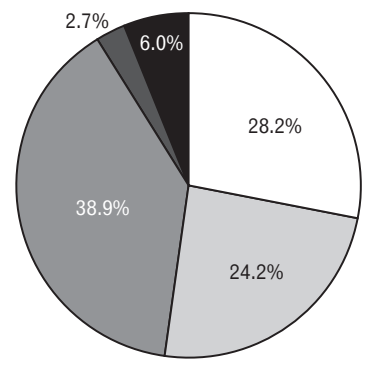

Abnormal delayed gastric scintigraphy

Presence of gastroparetic symptoms for more than

3 months plus a physician's diagnosis of gastroparesis

Presence of gastroparetic symptoms more than

3 months plus food retention on gastroscopy or upper GI study

Own definition

Not sure

C

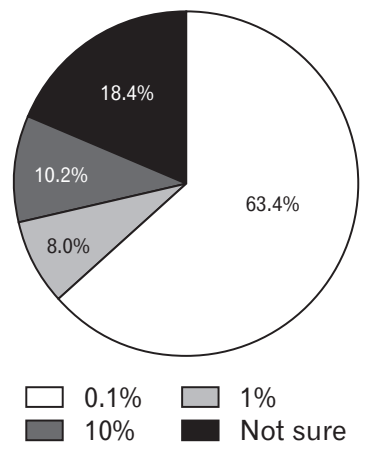

B

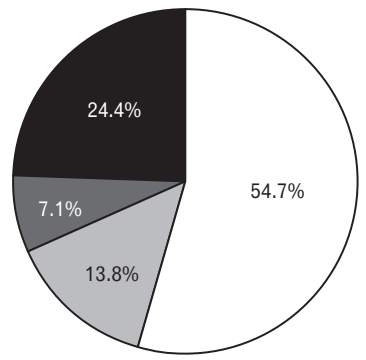

Gastric emptying scintigraphy

Stable isotope breath test

Wireless motility capsule

Not sure

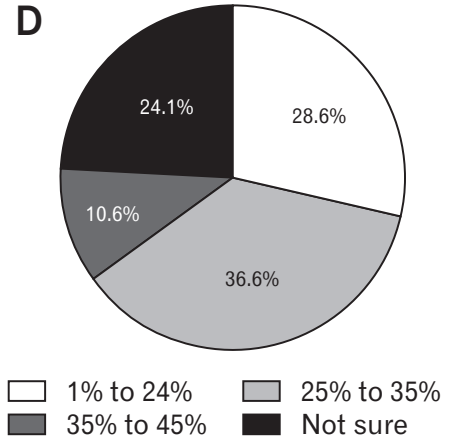

Figure 1. Distribution of knowledge for gastroparesis in Asia. (A) "What is your definition of gastroparesis?" and (B) "What is the gold-standard for gastroparesis?”. Real-world experience for gastroparesis in Asia (C) "How common is gastroparesis after 10 years of diagnosis of type 2 diabetes mellitus in your practice?" and (D) "What is the proportion of functional dyspepsia patients who may suffer from delayed gastric emptying in your practice?" GI, gastrointestinal.
Asian doctors. The "presence of gastroparesis symptoms for more than 3 months and food retention on gastroscopy or upper GI study" was the most common definition (38.9\%), and this was fol- lowed by an "abnormal delayed gastric scintigraphy" (28.2\%) and the "presence of gastroparesis symptoms for more than 3 months and a physician's diagnosis of gastroparesis" (24.2\%) (Fig. 1A). Fif- 
ty-five percent of Asian doctors in this survey thought that the gold standard of diagnosis for gastroparesis is GES (Fig. 1B). However, almost a quarter $(24.4 \%)$ of the respondents were not sure of the gold-standard test, suggesting that the diagnosis of gastroparesis is not familiar to Asian doctors.

\section{Real-world Experience for Gastroparesis in Asia}

In Asian clinical practice, the prevalence of gastroparesis after 10 years of diagnosis of type 2 diabetes mellitus was relatively low, ac-

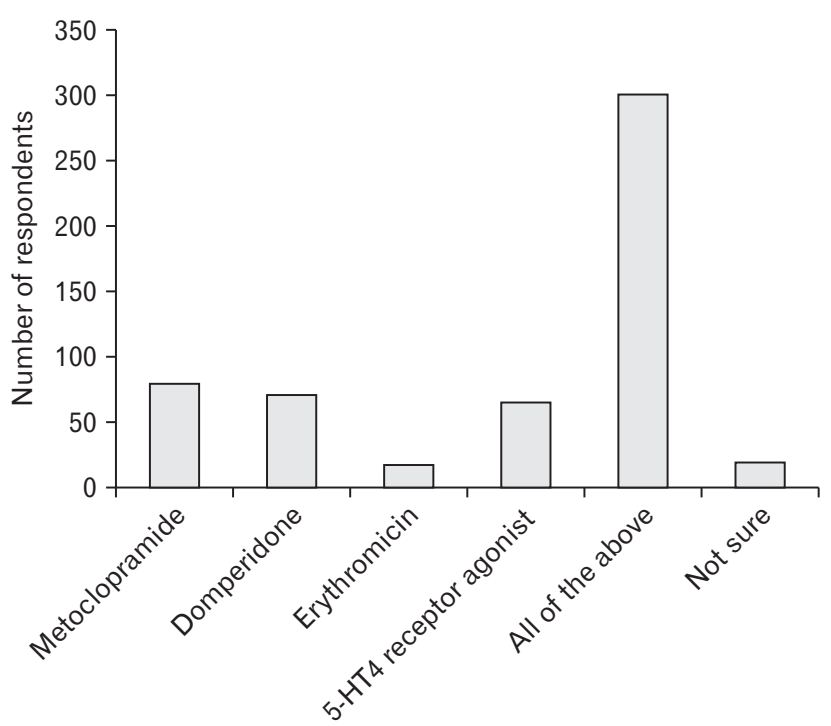

Figure 2. Drug that used for gastroparesis in Asia. 5- $\mathrm{HT}_{4}, 5$-hydroxytryptamine receptor 4 .

A Do you think it is important to diagnose gastroparesis?
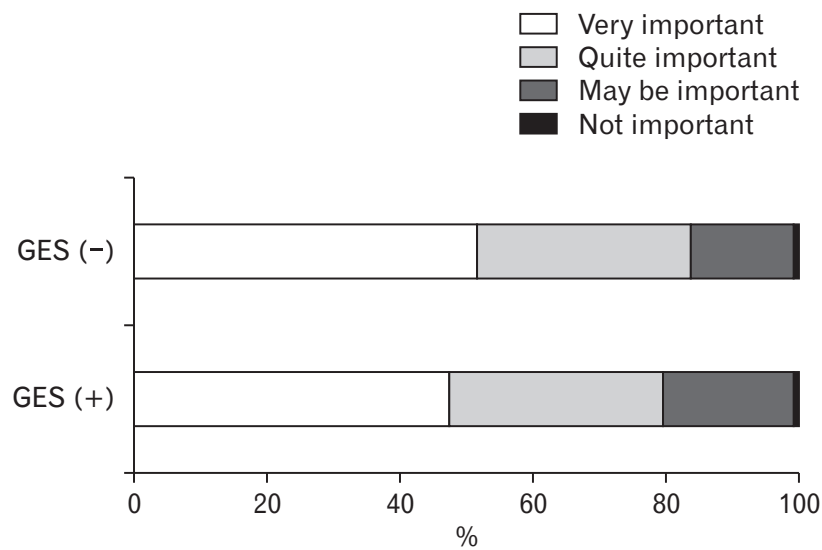

cording to this survey. Over two-thirds of doctors answered that they experienced only $0.1 \%$ of gastroparesis in patients with diabetes mellitus (Fig. 1C). In contrast, patients with FD more commonly suffer from delayed gastric emptying compared with type 2 diabetes mellitus. Almost half of the respondents suspect that 25 to 45 percent of their FD patients may be suffering from gastroparesis (Fig. 1D). When Asian doctors manage patients with suspected gastroparesis, they were more likely to start drug treatment than to order relevant investigation ( $58.3 \%$ vs $38.7 \%$ ). Various prokinetic drugs, including metoclopramide (16.1\%), domperidone (14.3\%), erythromycin (3.5\%), 5-hydroxytryptamine receptor 4 agonist (13.3\%), were being used for the treatment of gastroparesis. Most doctors used all of these drugs alternatively (Fig. 2).

\section{The Attitude of Asian Doctors Towards Gastroparesis}

Despite experiencing a low number of patients in Asia, the majority of doctors thought that the diagnosis of gastroparesis was very important $(48.8 \%)$ or quite important $(32.0 \%)$. The main reason why Asian doctors were not interested in diagnosing gastroparesis was "the lack of reliable diagnostic tests" (46.8\%) or "a lack of effective treatment" (41.5\%). When it was analyzed according to GES availability, the importance of the diagnosis of gastroparesis was not significantly different $(P=0.697)$ (Fig. 3A). However, the reason for low interest in diagnosing gastroparesis was significantly different between doctors who available GES and doctors who are not available GES $(P<0.01)$ (Fig. 3B). Amongst doctors with access to GES, their main reason for low interest in diagnosing gastropa-

B What do you think is the reason for lack of interest in gastroparesis diagnosis?

\section{No available reliable test} No available effective treatment

$\square$ It is not the cause of gastroparetic symptoms

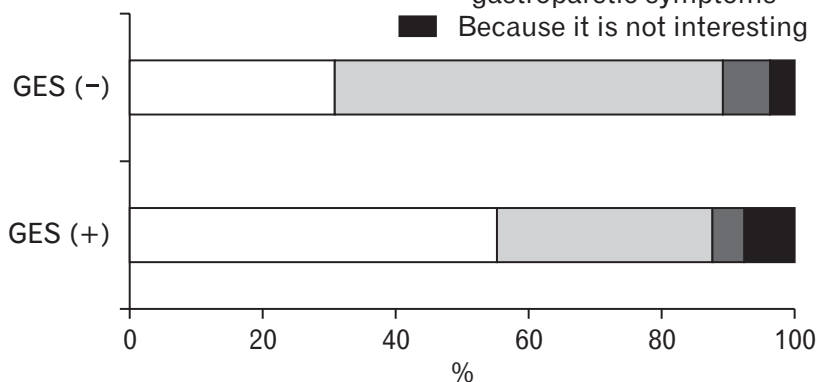

Figure 3. The difference in answer to the questions (A) "Do you think it is important to diagnose gastroparesis? and (B) "What do you think is the reason for lack of interest in gastroparesis diagnosis?” according to gastric emptying scintigraphy (GES) availability $(P=0.697$ and $P<0.01$ for $(\mathrm{A})$ and (B), respectively. Chi-square test between GES [-] and GES [+]). 
resis was "a lack of effective treatment." In contrast, most doctors who had no access to GES chose "a lack of reliable diagnostic tests" as their reason for low interest in diagnosing gastroparesis.

\section{Management of Gastroparesis}

Most doctors had never diagnosed gastroparesis at all (25.2\%) or diagnosed fewer than 5 patients a year (52.1\%). Two-thirds of doctors preferred a GES to diagnose gastroparesis. However, only $41.7 \%$ of respondents had access to a GES in their institute. Among all of the participants, $65.1 \%$ did not order GES in the last year. Multivariate analysis demonstrated that the most critical factor for ordering a GES was the availability of a GES in their institute. Country location and specialty type were additional factors in ordering a GES (Table 3).

There were many differences in the performance of GES among institutes and countries in terms of diagnostic criteria for delayed gastric retention (GE), time frame of GES, and test meal. About a quarter of the respondents used $10.0 \%$ retention at 4 hours for the determination of delayed $\mathrm{GE}$, but more institutions used a higher retention rate (15-20\%) at 4 hours in Asia (Fig. 4A). Twothirds of respondents did not know the time frame of GES in their institute. The 4-hour method is the most common time frame, but many institutes used a shorter 2-hour method which is less sensitive for the detection of delayed GE (Fig. 4B). ${ }^{14}$ The noticeable difference in the protocol was the test meal. Although two-thirds of the respondents did not know what kind of test meal was used for GES in their institutions, a typical western test meal (2 slices of bread, strawberry jam, scrambled egg, and water) was most commonly used. Interestingly, a rice-based test meal (curry rice, rice with microwaved egg, and rice roll in laver) was also widely used in Asia (Fig. 4C).

\section{Discussion}

Gastroparesis is a major GI condition. However, a substantial proportion of respondents in this Asian multi-national survey was unable to give the correct definition and correct diagnostic test. The

Table 3. Multivariate Analysis for Factors Affecting Ordering Gastric Emptying Scintigraphy

\begin{tabular}{lcccc}
\hline \multicolumn{1}{c}{ Variable } & Univariate OR $(95 \% \mathrm{CI})$ & $P$-value & Multivariate OR $(95 \% \mathrm{CI})$ & $P$-value \\
\hline Country (other/Japan) & $9.01(4.70-17.30)$ & $<0.001$ & $5.88(2.59-13.30)$ & $<.18(1.95-8.98)$ \\
Occupation (gastroenterologist/other) & $3.03(1.70-5.39)$ & $<0.01$ & & $<0.001$ \\
Years $(>10 / \leq 10 \mathrm{yr})$ & $1.15(0.76-1.74)$ & 0.50 & 0.45 & \\
Gender (male/female) & $0.85(0.56-1.30)$ & 0.25 & \\
Importance $(+/-)^{\mathrm{a}}$ & $1.33(0.82-2.17)$ & $<0.01$ & $36.50(20.10-66.50)$ & $<0.001$ \\
Availability $(+/-)$ & $44.00(24.80-78.10)$ & &
\end{tabular}

"Question of "Do you think it is important to diagnose gastroparesis."

GES, gastric emptying scintigraphy.

A

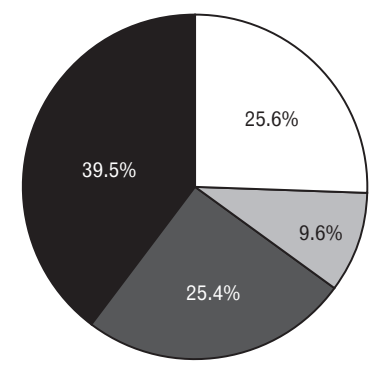

$>10 \%$ at $4 \mathrm{hr}$

$>20 \%$ at $4 \mathrm{hr}$
B

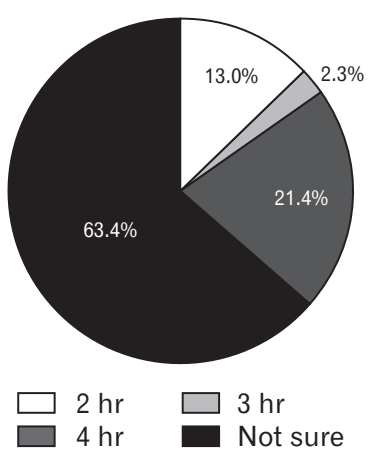

C

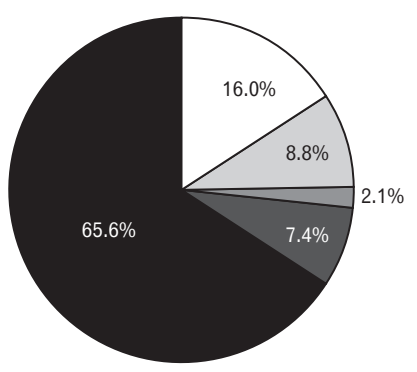

2 Slices of bread, strawberry jam, scrambled egg and water

$\square$ A bowl of rice with microwaved egg

$\square$ A plate of curry rice

$\square$ Customized meal*

- Not sure

Figure 4. Real-world management of gastroparesis in Asia (A) "In your institute, delayed GE (gastric retention) was determined to be," (B) "What kind of time frame is your hospital doing gastric emptying scan?," and (C) "What kind of test meal for gastric emptying scan is your hospital using?" *Noodles and meat, Gimbap (Korean rice rolled in dried laver) with or without egg, liquid food, microwaved egg without rice, and oatmeal. 
level of poor recognition among the respondents is alarming. Consequently, more than a quarter of respondents had never diagnosed any gastroparesis in the last year. These findings confirmed our fear that Asian patients with gastroparesis may be under-diagnosed. However, another reason for the low rate of diagnosis may be due to a lack of GES facility, variation of study methods and results interpretation. The majority (80.8\%) of responders perceived that diagnosing gastroparesis was quite or very important. The main reason for not ordering GES may due to a lack of reliable tests in the respondent's institution. This lack of reliable test may additionally explain why $58.3 \%$ of respondents would start drug treatment first instead of investigation for suspected patients with gastroparesis. Prokinetics are the first choice; in this case, they can be secondary to the varieties of prokinetics and availability as over-the-counter drugs in Asia. Furthermore, GES may not be covered by healthcare insurance systems in certain Asian countries like Japan.

However, there is still disagreement regarding the standard of the gastric emptying test. It has been more than 10 years since the publication of the Society of Nuclear Medicine and Molecular Imaging guidelines on solid meal gastric emptying scan. ${ }^{15}$ Recently, a follow-up study in the United States (US) showed that 69.3\% of protocols were not compliant with the content or preparation of the consensus meal. ${ }^{16}$ In Asia, literature review showed that there were 3 different types of test meals reported. In Thailand, Vasavid et $\mathrm{al}^{17}$ used $100 \mathrm{~g}$ steamed rice, a microwaved labelled egg, and 100 $\mathrm{mL}$ water. The total calorie of the meal was $267 \mathrm{kcal}$. The sauce, which had soy sauce and salt as the main ingredients, was provided to volunteers to improve the meal taste. ${ }^{17}$ In Taiwan, Tseng et al ${ }^{18}$ used instant oatmeal mixed with $1 \mathrm{mCi}$ of ${ }^{99 \mathrm{~m}} \mathrm{Tc}$ diethylenetriaminepentacetic acid. In India, Anudeep et $\mathrm{al}^{19}$ also performed a study on gastroparesis, the test meal comprised of 3 idlis prepared from 60 $\mathrm{g}$ of MTR premixed rice idli formula (MTR Foods, Bengaluru, India) and $60 \mathrm{~mL}$ water. The total caloric content of the meal was $215 \mathrm{kcal}$ and the fat content was $3 \%$ by weight. The participant had to consume the meal within 10 minutes with $10 \mathrm{~g}$ of coconut chutney. Inconsistent or different GES protocols impaired standardized reporting, decreased agreement, and impaired research sharing by clinicians. $^{19}$

Our survey showed that our respondents may not recognize patients with a high risk of gastroparesis. However, this may not be unique to Asian countries. In an online survey of gastroparesis patients in the US, Yu et $\mathrm{al}^{20}$ reported that most respondents were diagnosed 5 years after the symptoms began, and $50 \%$ were either somewhat dissatisfied or dissatisfied with their medical care. A recent population-based study in the United States using Explorys
(IBM, Cleveland, USA), an electronic medical records database with International Classification of Diseases-9 codes indexed diagnoses, estimated the proportion of study population with gastroparesis. The results showed that out of a total of 43827910 medical records, $69950(0.2 \%)$ had a diagnosis of gastroparesis recorded. The authors also observed concurrent gastroparesis in $4.6 \%$ medical records with type 1 diabetes mellitus, $1.3 \%$ medical records with type 2 diabetes mellitus, and $0.05 \%$ medical records with no history of any potential etiologies of gastroparesis. ${ }^{21}$ The proportion of type 2 diabetes mellitus with gastroparesis symptoms who had delayed gastric emptying ranged from $13.8 \%$ to $29.0 \%$ in Asian studies. 8-23\% of Asian people suffer from FD. ${ }^{22,23}$ The estimated proportion of Asian FD patients with delayed gastric emptying was $13.3 \% .^{17-19}$

\section{Future of Gastroparesis in Asia}

Both diabetes mellitus and chronic upper GI symptoms are prevalent in Asia. ${ }^{22,24}$ Population-based studies on the prevalence, clinical features, severity, and treatment response of gastroparesis in Asia are lacking. The lack of effective therapy in the past may have influenced a negative attitude towards gastroparesis. This ANMA gastroparesis survey seems to confirm this perception amongst Asian gastroenterologists and general physicians. However, the current situation with gastroparesis may eventually follow that of achalasia, another upper GI dysmotility disease. The advent of better diagnostic modalities such as high-resolution manometry and effective minimally invasive endoscopic therapy, peroral endoscopic myotomy (POEM), has led to an increased awareness of achalasia in Asia, and globally, in the last few years. ${ }^{25}$ Similarly, with the growing reports of promising pharmacological and non-pharmacological therapy, together with concerted efforts by Asian neurogastroenterology experts to standardize diagnostic methods, it is hopeful that increased awareness to diagnose and treat gastroparesis in Asia will happen in the not too distant future.

Gharibans et al ${ }^{26}$ performed non-invasive gastric electrical mapping in patients with FD and gastroparesis. Cutaneous high-resolution electrogastrography is a non-invasive method that can detect spatial features of the gastric slow wave. Studies showed that $44 \%$ of patients with foregut symptoms had spatial slow-wave abnormalities. Subjects with a higher percentage of slow waves with aberrant propagation direction also had a more severe abdominal pain and higher total GCSI score. ${ }^{26}$ In a meta-analysis by Vijayvargiya et al, ${ }^{27}$ they found prokinetic agents to significantly accelerate GE (when optimal test methods were used) and to produce significant improvements in upper GI symptoms. ${ }^{27}$ Other newer agents include 
antiemetics (aprepitant), and fundic relaxants (acotiamide and buspirone). ${ }^{1,12}$ Further validation is required for the clinical use. Gastric POEM first described by Khashab et $\mathrm{al}^{28}$ in 2013 is fast becoming a treatment option for refractory gastroparesis especially for symptoms of nausea and vomiting. ${ }^{29}$ Mekaroonkamol et $\mathrm{al}^{29}$ compared refractory gastroparesis patients who underwent gastric POEM and those without, and they found the procedure significantly improved symptoms and quality of life, as well as reduced health care use related to gastroparesis.

\section{Conclusion}

In conclusion, gastroparesis can be challenging to diagnose due to the lack of instrument, standardized method, and paucity of research data on normative value, risk factors, and treatment studies in Asian patients. Our survey confirmed our suspicion but also showed areas of improvement needed to better manage gastroparesis and what needs to be done to improve the care of gastroparesis patients in Asia. Future strategies should concentrate on how to disseminate the latest knowledge of gastroparesis in Asia. In particular, there is an urgent need to estimate the magnitude of the problems in high risk and idiopathic patients as well as a standardized diagnostic procedure in Asia.

\section{Financial support: None.}

\section{Conflicts of interest: None.}

Author contributions: Ideas formulation of ideas, research goals and aims, development or design of methodology: Kewin T H Siah, Tadayuki Oshima, and Yong Sung Kim; data curation and formal analysis: Tadayuki Oshima and Yong Sung Kim; conducting a research and investigation process, specifically performing the experiments, or data/evidence collection: Sanjiv Mahadeva, Hyojin Park, Min-Hu Chen, Ching-Liang Lu, Xiaohua Hou, Duc T Quach, Ari F Syam, M Masudur Rahman, Yinglian Xiao, Liu Jinsong, and Andrew S B Chua; oversight and leadership responsibility for the research activity planning and execution, including mentorship external to the core team: Hiroto Miwa; creation and/ or presentation of the published work, specifically writing the initial draft: Kewin T H Siah, Tadayuki Oshima, Yong Sung Kim; and critical review, commentary, or revision: Tanisa Patcharatrakul and Chien-Lin Chen.

\section{References}

1. Camilleri M, Chedid V, Ford AC, et al. Gastroparesis. Nat Rev Dis Primers 2018;4:41.

2. Tack J, Carbone F. Functional dyspepsia and gastroparesis. Curr Opin Gastroenterol 2017;33:446-454.

3. Pasricha PJ, Camilleri M, Hasler WL, Parkman HP. White paper AGA: gastroparesis: clinical and regulatory insights for clinical trials. Clin Gastroenterol Hepatol 2017;15:1184-1190.

4. Liu N, Abell T. Gastroparesis updates on pathogenesis and management. Gut Liver 2017;11:579-589.

5. Kim BJ, Kuo B. Gastroparesis and functional dyspepsia: a blurring distinction of pathophysiology and treatment. J Neurogastroenterol Motil 2019;25:27-35.

6. Quartero AO, de Wit NJ, Lodder AC, Numans ME, Smout AJ, Hoes AW. Disturbed solid-phase gastric emptying in functional dyspepsia: a meta-analysis. Dig Dis Sci 1998;43:2028-2033.

7. Lacy BE, Everhart K, Crowell MD. Functional dyspepsia: clinical symptoms, psychological findings, and GCSI scores. Dig Dis Sci 2019;64:1281-1287.

8. Talley NJ, Ford AC. Functional dyspepsia. N Engl J Med 2015;373:1853-1863

9. Moayyedi P, Lacy BE, Andrews CN, Enns RA, Howden CW, Vakil $\mathrm{N}$. ACG and CAG clinical guideline: management of dyspepsia. Am J Gastroenterol 2017;112:988-1013.

10. Miwa H, Ghoshal UC, Gonlachanvit S, et al. Asian consensus report on functional dyspepsia. J Neurogastroenterol Motil 2012;18:150-168.

11. Asano H, Tomita T, Nakamura K, et al. Prevalence of gastric motility disorders in patients with functional dyspepsia. J Neurogastroenterol Motil 2017;23:392-399.

12. Grover M, Farrugia G, Stanghellini V. Gastroparesis: a turning point in understanding and treatment. Gut 2019;68:2238-2250.

13. Wuestenberghs F, Juge M, Melchior C, Desprez C, Leroi AM, Gourcerol G. Association between symptoms, quality of life, and gastric emptying in dyspeptic patients. J Neurogastroenterol Motil 2019;25:534543

14. Abell TL, Camilleri M, Donohoe K, et al. Consensus recommendations for gastric emptying scintigraphy: a joint report of the American Neurogastroenterology and Motility Society and the Society of Nuclear Medicine. Am J Gastroenterol 2008;103:753-763.

15. Abell TL, Camilleri M, Donohoe K, et al. Consensus recommendations for gastric emptying scintigraphy: a joint report of the American Neurogastroenterology and Motility Society and the Society of Nuclear Medicine. J Nucl Med Technol 2008;36:44-54.

16. Farrell MB, Costello M, McKee JD, Gordon LL, Fig LM. Compliance with gastric-emptying scintigraphy guidelines: an analysis of the intersocietal accreditation commission database. J Nucl Med Technol 2017;45:6-13

17. Vasavid $P$, Chaiwatanarat T, Pusuwan $P$, et al. Normal solid gastric emptying values measured by scintigraphy using asian-style meal: a multicenter study in healthy volunteers. J Neurogastroenterol Motil 2014;20:371- 
378.

18. Tseng PH, Wu YW, Lee YC, et al. Normal values and symptom correlation of a simplified oatmeal-based gastric emptying study in the Chinese population. J Gastroenterol Hepatol 2014;29:1873-1882.

19. Anudeep V, Vinod KV, Pandit N, et al. Prevalence and predictors of delayed gastric emptying among Indian patients with long-standing type 2 diabetes mellitus. Indian J Gastroenterol 2016;35:385-392.

20. Yu D, Ramsey FV, Norton WF, et al. The burdens, concerns, and quality of life of patients with gastroparesis. Dig Dis Sci 2017;62:879-893.

21. Syed AR, Wolfe MM, Calles-Escandon J. Epidemiology and diagnosis of gastroparesis in the united states: a population-based study. J Clin Gastroenterol 2020;54:50-54.

22. Ghoshal UC, Singh R, Chang FY, et al. Epidemiology of uninvestigated and functional dyspepsia in Asia: facts and fiction. J Neurogastroenterol Motil 2011;17:235-244.

23. Oshima T, Miwa H. Epidemiology of functional gastrointestinal disorders in Japan and in the world. J Neurogastroenterol Motil 2015;21:320329.

24. Ramachandran A, Snehalatha C, Shetty AS, Nanditha A. Trends in prevalence of diabetes in Asian countries. World J Diabetes 2012;3:110117.

25. Jung HK, Hong SJ, Lee OY, et al. 2019 Seoul consensus on esophageal achalasia guidelines. J Neurogastroenterol Motil 2020;26:180-203.

26. Gharibans AA, Coleman TP, Mousa H, Kunkel DC. Spatial patterns from high-resolution electrogastrography correlate with severity of symptoms in patients with functional dyspepsia and gastroparesis. Clin Gastroenterol Hepatol 2019;17:2668-2677.

27. Vijayvargiya P, Camilleri M, Chedid V, Mandawat A, Erwin PJ, Murad $\mathrm{MH}$. Effects of promotility agents on gastric emptying and symptoms: a systematic review and meta-analysis. Gastroenterology 2019;156:16501660 .

28. Khashab MA, Stein E, Clarke JO, et al. Gastric peroral endoscopic myotomy for refractory gastroparesis: first human endoscopic pyloromyotomy (with video). Gastrointest Endosc 2013;78:764-768.

29. Mekaroonkamol P, Dacha S, Wang L, et al. Gastric peroral endoscopic pyloromyotomy reduces symptoms, increases quality of life, and reduces health care use for patients with gastroparesis. Clin Gastroenterol Hepatol 2019;17:82-89. 